\title{
A new method combining discrete wavelet transform and neural network for high energy physics problem
}

\author{
Hind Khudheyer ${ }^{1}$, Hisham Jashami ${ }^{2}$ \\ ${ }^{1}$ University of Karbala, Department of physics, Karbala, Iraq \\ ${ }^{2}$ University of Karbala, Department of Civil Engineering, Karbala, Iraq
}

\begin{abstract}
In this paper, a new method proposed for high energy physics problem combining discrete wavelet transform and neural network. DWT used to decrease the size of features and the output of DWT wired to the neural network. The neural network applied to classify the features that obtained by DWT. Several statistical parameters are calculated to evaluate the performance of the proposed method. The proposed method presented remarkable results when compared with previous studies. @ 2019 ijrei.com. All rights reserved
\end{abstract}

Keywords: SVM, High-Energy Physics, Power of Signal

\section{Introduction}

High energy physics data examination is an honest multivariate problem. Notwithstanding the detail that multivariate methods have remained applied in High Energy Physics for an extended period, the volatile development of machine learning (ML) methods throughout the previous two decades had lone an incomplete influence on the familiar chic in which data investigation is achieved in this arena. Lone lately an augmented attention in the ML knowledge learned in other ranges of discipline can be experiential.

ML applied to different fields successfully such as face recognition, image classification, and data classification [1]. Then, in recent years' number of studies presented which applied ML to high energy physics. Because the high energy physics is difficult and hot topic exactly in the last years, then, we developed software has ability to classify the high energy physics problem in low computation time and high accurate.

\section{Material \& Methods}

\subsection{Discrete Wavelet Transform (DWT)}

The discrete wavelet transform (DWT) is an application of the wavelet transform (WT) using a discrete set of the wavelet scales and conversions submitting some definite rules. On other hand, this transmute decays the signal into equally orthogonal set of wavelets, which is the chief variance from the continuous wavelet transform (CWT), or its application for the discrete time series occasionally called discrete-time continuous wavelet transform (DT-CWT) [2][1]. The process of DWT presented in Figure 1.

$$
D W T(i, k)=\frac{1}{\sqrt{a_{0}^{j}}}=\sum_{n=-\infty}^{\infty} f(n) \psi\left(\frac{\left(n-a_{0}^{j} k b_{0}{ }^{*}\right)}{a_{0}^{j}}\right)
$$

Where $j, k, n \in \mathrm{Z}$ and $a_{0}>1$

\subsection{Neural Network}

ANN is a data treating model that is stimulated by the way biological nervous organizations, like the brain, procedure data. The key component of this model is the original organization of the data treating organization. It is collected of a great amount of extremely organized processing rudiments employed in agreement to resolve exact difficulties. ANN, like individuals, learn by instance. 


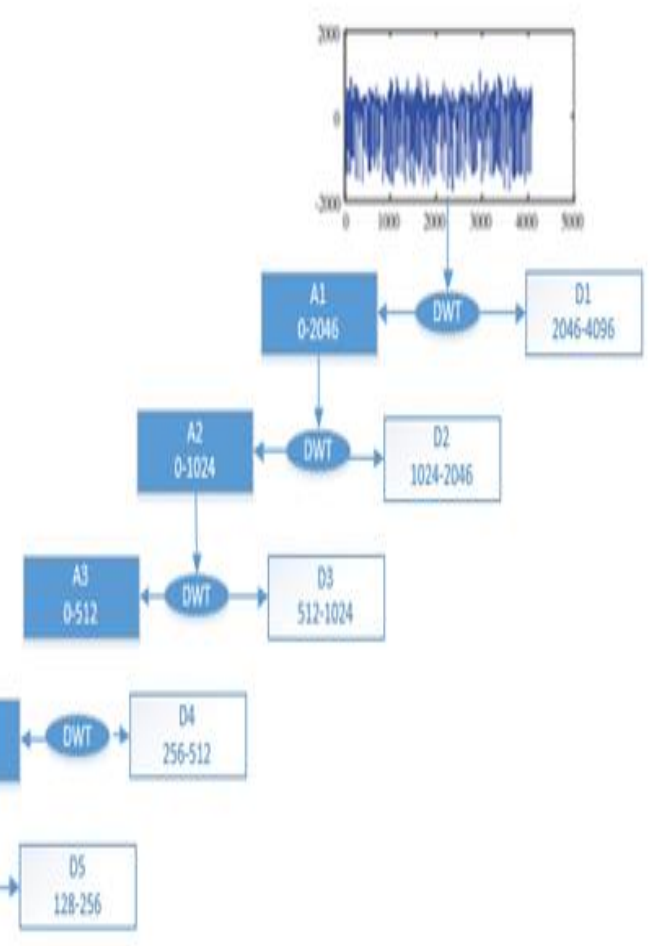

Figure 1: DWT Process [4].

An ANN is arranged for an exact presentation, like pattern classification or data processing, over a learning procedure. Knowledge in biological schemes includes changes to the synaptic influences that occur among the neurons. The neural network mathematical model represented in Equation (2).

$$
f(x)=\mathrm{b}+\sum_{i=0}^{n} x_{i} w_{i}
$$

Where $\mathrm{n}$ is number of examples, $\mathrm{b}$ is the basis, $\mathrm{i}$ is the loop that start from 0 to $n, x$ is the input features, $w$ is the weight [3-5]. See the simple neural network architecture in Figure 2.

First column is the lesson label (1 for signal, 0 for background), shadowed by the 27 regularized features (22 low-level features then 5 high-level features).

\subsection{Proposed Method}

In this section, the data first read and then DWT applied to mine essential features from records that consist. The features calculated by using the Equation (1). Then, several DWT parameters are calculated such as (A1, A2, A3, D1, D2, and D3). Furthermore, data separate into two sets training and testing. Generally, the system consists from number of station which in the first stage the data read. Then, calculate the DWT for each row data and divide the data into training and testing sets. The neural network trained by using the training sets after training complete the NN tested by testing set and the accuracy will be calculated. The process of the proposed method shown in Figure 3.

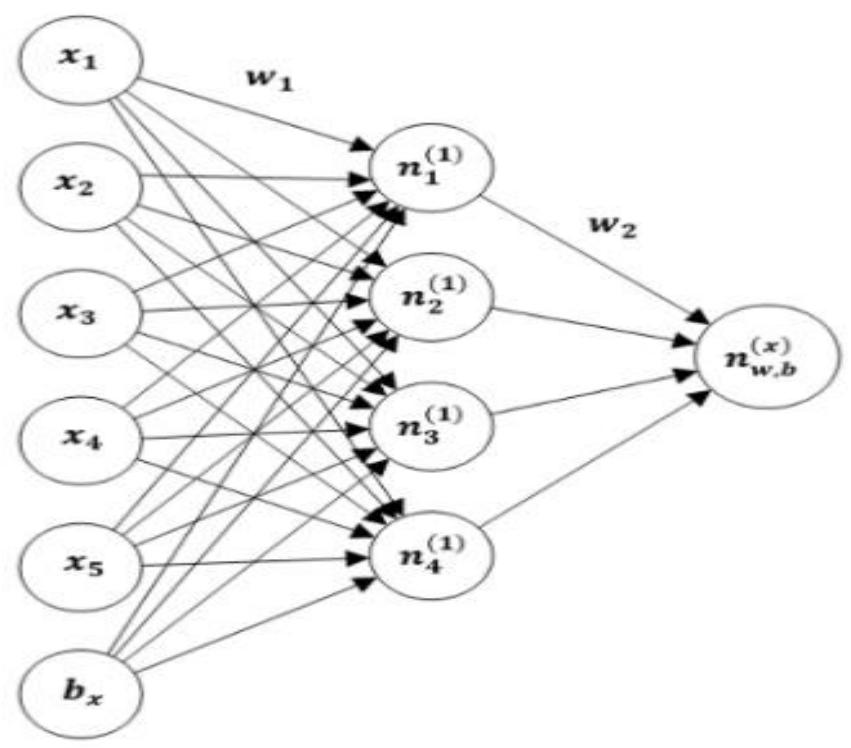

Figure 2: Neural network architecture

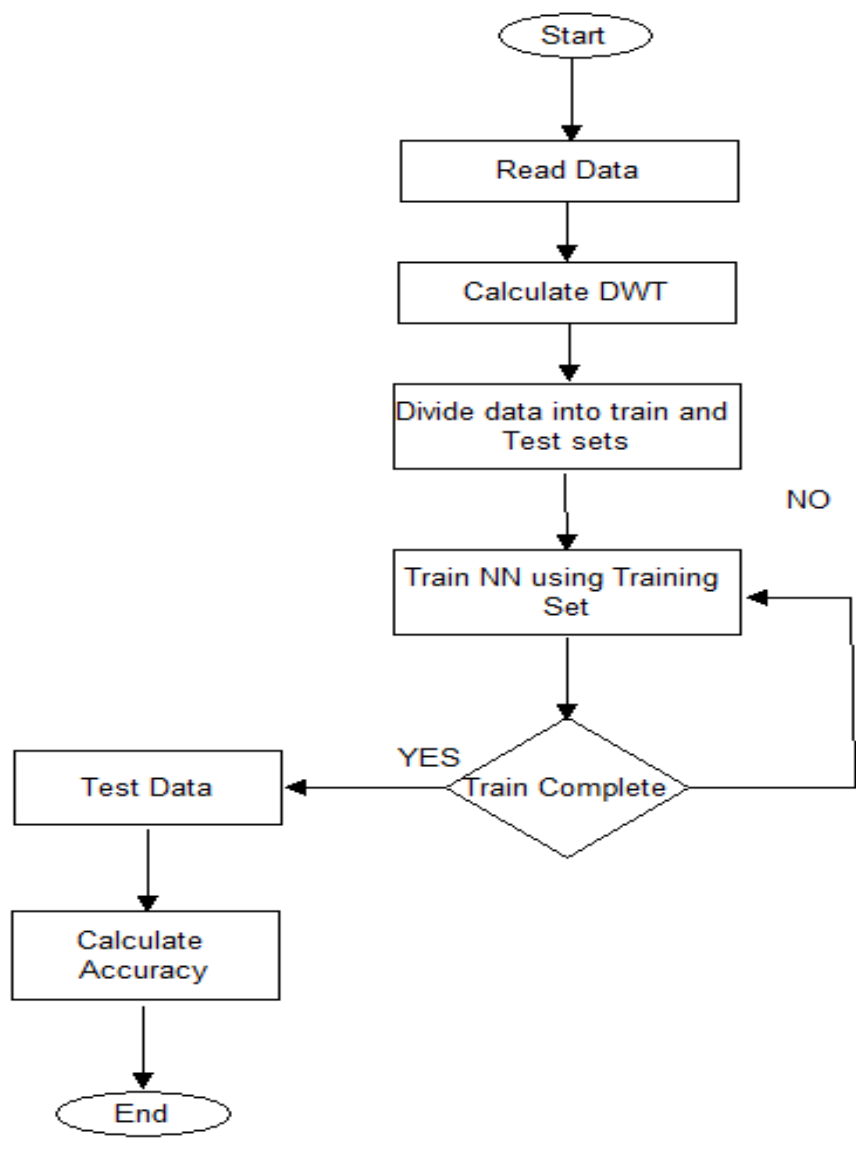

Figure 3: Proposed Method Structure 


\section{Results and Discussion}

The experimental results achieved by using MATLAB2018 by applying cross 10 to avoid the overfitting issue. Several parameters are calculated to evaluate the performance of the proposed method and the results listed in Table 1.

Table 1: Experimental Results

\begin{tabular}{|c|c|}
\hline Parameter & Proposed Method \\
\hline Sensitivity & 1.0000 \\
\hline Sensitivity & 0.8850 \\
\hline Specificity & 0.9901 \\
\hline Accuracy & 0.9921 \\
\hline
\end{tabular}

Then, our study compared with best previous studies in this field and show that our method presented remarkable results see Table 2 .

Table 2: Results Comparison

\begin{tabular}{|c|c|}
\hline ref & Results \\
\hline Hind et al.[7] & 0.9893 \\
\hline Our Study & 0.9921 \\
\hline
\end{tabular}

\section{Conclusion}

In this study remarkable results obtained compared to previous studies. The DWT extracted high level and sensitive features in low processing time. This lead to obtain accurate results in low processing time when compared with well-known researches in this field.

As feature work the DWT can apply to same field by combining with other classifiers. Furthermore, other signal processing functions can be applied to the same field.

\section{References}

[1] Karim, A. M., Güzel, M. S., Tolun, M. R., Kaya, H., and Çelebi, F. V., A New Generalized Deep Learning Framework Combining Sparse Autoencoder and Taguchi Method for Novel Data Classification and Processing, Mathematical Problems in Engineering, pp. 1-13, 2018.
[2] A. M. Karim, Ö. Karal, and F. V Çelebi, "A New Automatic Epilepsy Serious Detection Method by Using Deep Learning Based on Discrete Wavelet Transform," no. 4, pp. 15-18, 2018.

[3] E. Yavuz, M. C. Kasapbaşı, C. Eyüpoğlu, and R. Yazıcı, An epileptic seizure detection system based on cepstral analysis and generalized regression neural network, biocybernetics and biomedical engineering, vol. 38, no. 2, pp. 201-216, 2018.

[4] R. Djemili, H. Bourouba, and M. C. Amara Korba, "Application of empirical mode decomposition and artificial neural network for the classification of normal and epileptic EEG signals," Biocybernetics and Biomedical Engineering, vol. 36, no. 1, pp. 285-291, 2016.

[5] Karim, A. M., Güzel, M. S., Tolun, M. R., Kaya, H., \& Çelebi, F. V. (2019). A new framework using deep auto-encoder and energy spectral density for medical waveform data classification and processing. Biocybernetics and Biomedical Engineering, 39(1), 148-159. doi:10.1016/j.bbe.2018.11.004

[6] A. M. Karim, Ö. Karal, and F. V Çelebi, "A New Automatic Epilepsy Serious Detection Method by Using Deep Learning Based on Discrete Wavelet Transform," no. 4, pp. 15-18, 2018.

[7] Hind Khudheyer, Hisham Jashami, Support vector machine based on power of signal for solving high-energy physics problem, International journal of research in engineering and innovation (IJREI), vol 3, issue 1 (2019), 57-60.

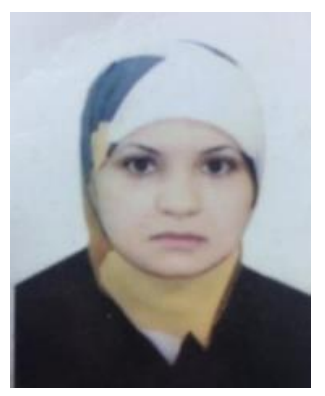

Hind Khudheyer Abbas is a lecturer at University of Karbala, Iraq, in where she was born in Karbala. In 2005, she received her B.Sc. degree in physics from Baghdad University in Baghdad, Iraq. She obtained M.Sc. degree in physics science from Ankara University in Ankara, Turkey, in 2012.

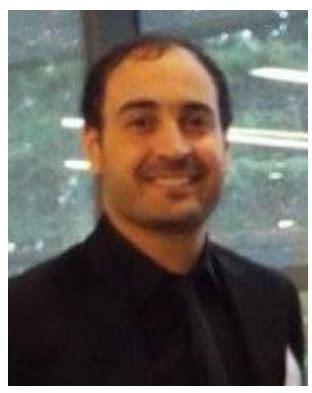

Hisham Jashami obtained his Master of Science in Civil Engineering from Gaziantep University, Turkey in 2012. He is from Iraq, where he completed his undergraduate in the same area from Al-Mustansiriya University in 2006.

Cite this article as: Hind Khudheyer, Hisham Jashami, A new method combining discrete wavelet transform and neural network for high energy physics problem, International journal of research in engineering and innovation (IJREI), vol 3, issue 2 (2019), 79-81. https://doi.org/10.36037/IJREI.2019.3201 